\title{
Associated forward photon-jet production as a probe of the nuclear unintegrated glue
}

Wolfgang Schäfer*

Institute of Nuclear Physics PAN, Kraków, Poland

E-mail: Wolfgang.Schafereifj.edu.pl

\begin{abstract}
To the lowest order in $\mathrm{PQCD}$, the underlying subprocess for hard isolated photon production in the proton fragmentation region of $p p$ and $p A$ collisions is the QCD Compton effect $q^{*} g \rightarrow q \gamma$, where $q^{*}$ is the valence quark in the beam proton. In the $q^{*} p$ collisions frame, the $q g$ dijet acoplanarity momentum $\vec{\Delta}$ comes from the exchanged gluon and the $\vec{\Delta}$-distribution measures the unintegrated gluon density in the target nucleon. Both the incident and produced quarks undergo multiple gluon exchanges in an extended nuclear target; in all other dijet processes that entails a complex nonlinear $k_{\perp}$-factorization for the dijet spectrum. We demonstrate that the $q \gamma$ dijet production is an exceptional reaction for which the linear $k_{\perp}$-factorization holds, which makes it a unique direct probe of the collective nuclear gluon density at small- $x$. We discuss properties of the nuclear unintegrated gluon distribution, and derive a relationship between the nuclear broadening of the acoplanarity momentum distribution and the nuclear saturation scale.
\end{abstract}

XVIII International Workshop on Deep-Inelastic Scattering and Related Subjects, DIS 2010 April 19-23, 2010

Firenze, Italy

\footnotetext{
* Speaker.
} 


\section{Dipole scattering amplitude defines the unintegrated glue}

Small- $x$ hard processes, like deep inelastic scattering on a heavy, opaque, nucleus differ in an essential way from their counterparts on the free nucleon. The reason being, that for strongly absorbing targets, diffractive processes, where the target is left intact(!), will make up for $50 \%$ of all deep inelastic interactions [1]. Recent calculations [2] for realistic nuclei find a still substantial $\sim 20 \div 30 \%$ ratio of diffractive to inclusive DIS.

The presence of diffractive final states is, via unitarity, intimately related to nuclear shadowing of nuclear structure functions. Large shadowing corrections however imply, that we must give up the standard treatment of deep inelastic scattering in terms of parton densities which involve two partons in the $t$-channel of the forward Compton amplitude.

For example, the parton phenomenology at small $-x$ is based on the unintegrated gluon density of the target

$$
f(x, \vec{p})=\frac{4 \pi \alpha_{S}}{N_{c}} \frac{1}{\vec{p}^{4}} \frac{\partial G\left(x, \vec{p}^{2}\right)}{\partial \log (\vec{p})},
$$

where $\vec{p}$ is the gluon's transverse momentum and $x$ is the relevant Bjorken variable. The formalism which relates the unintegrated gluon distribution to physical observables is the (linear) $k_{\perp}$-factorization.

We can generalize the concept of an unintegrated gluon distribution including multiple scattering effects by means of the Fourier-transform of the scattering amplitude $\Gamma(\vec{b}, x, \vec{r})$ of a color dipole of size $\vec{r}$ at impact parameter $\vec{b}$ :

$$
\int \frac{d^{2} \vec{r}}{(2 \pi)^{2}} \Gamma(\vec{b}, x, \vec{r}) e^{-i \vec{p} \vec{r}}=\left(1-w_{0}(\vec{b}, x)\right) \delta^{(2)}(\vec{p})-\phi(\vec{b}, x, \vec{p}),
$$

Here $\phi(\vec{b}, x, \vec{p})$ has the meaning of the $\vec{b}$-dependent unintegrated glue of a nucleus. For the explicit form of $w_{0}$, see e.g. [3]. After having included multiple scattering effects into the unintegrated glue, we have to give up the idea of universality of the unintegrated gluon distribution to a certain extent [3, 那. Namely, we cannot evaluate observables by simply plugging the above defined unintegrated glue into linear $k_{\perp}$-factorization formulas, but instead a new, nonlinear $k_{\perp}$-factorization emerges [3, 身.

Let us briefly review how the nuclear unintegrated glue is constructed from its free-nucleon counterpart: starting at moderately small $x \sim x_{A} \sim 0.01$, one can obtain the nuclear glue by plugging into eq.(1.2) the Glauber-Gribov form of the dipole amplitude:

$$
\Gamma\left(\vec{b}, x_{A}, \vec{r}\right)=1-\exp \left[-\sigma\left(x_{A}, \vec{r}\right) T_{A}(\vec{b}) / 2\right],
$$

where $T_{A}(\vec{b})$ is the nuclear matter density. The nuclear is obtained as an expansion over multiple convolutions of the free-nucleon unintegrated glue $f(x, \vec{p})[5]$ :

$$
\phi\left(\vec{b}, x_{A}, \vec{p}\right)=\sum w_{j}\left(v_{A}\right) f^{(j)}\left(x_{A}, \vec{p}\right) .
$$

Here

$$
f^{(j)}\left(x_{A}, \vec{p}\right)=\int\left[\prod^{j} d^{2} \vec{\kappa}_{i} f\left(x_{A}, \vec{\kappa}_{i}\right)\right] \delta^{(2)}\left(\vec{p}-\sum \vec{\kappa}_{i}\right)
$$




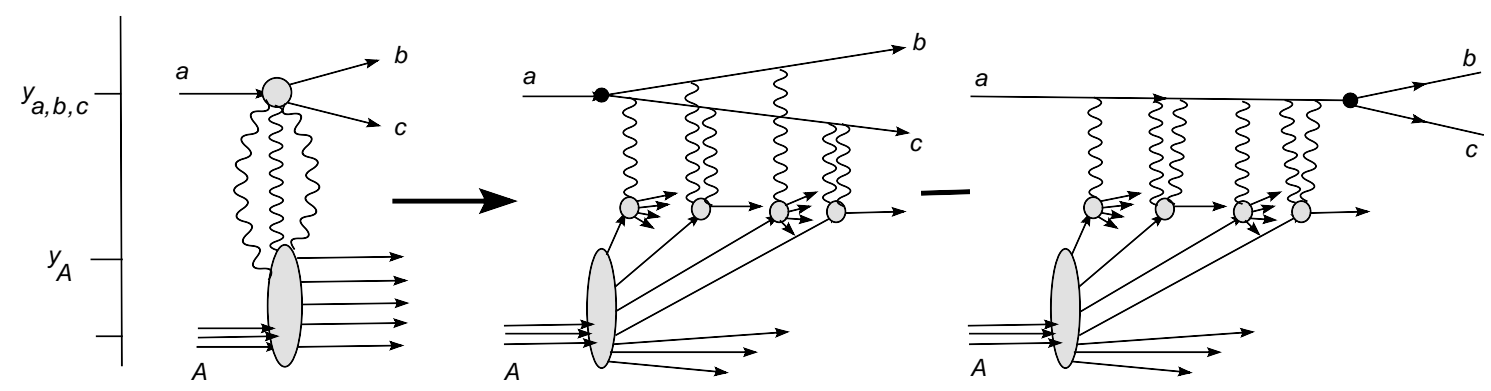

Figure 1: Dijet production as a breakup of a beam parton $a$ into its two-body $b c$ Fock component in the interaction with a nuclear target. Here we are interested in the case $a=q, b c=q \gamma$, where only the quark interacts with the nucleus through multigluon exchanges.

is the collective glue of $j$ overlapping nucleons, and

$$
v_{A}=v_{A}\left(\vec{b}, x_{A}\right)=\frac{1}{2} \alpha_{S}\left(q^{2}\right) \sigma_{0}\left(x_{A}\right) T_{A}(\vec{b}),
$$

is an effective opacity; the explicit form of the weights $w_{j}$ will again be found e.g. in [5], and $\sigma_{0}(x)=\int d^{2} \vec{p} f(x, \vec{p})$ is a nonperturbative parameter - the cross section of a large color dipole.

Using general properties of the large $-\vec{p}$ behaviour of $f$, one can derive the asymptotics of its multiple convolutions, and obtain the asymptotics of $\phi$ at transverse momenta much bigger than the saturation scale [5]:

$$
\phi\left(v_{A}, x_{A}, \vec{p}\right)=v_{A} f\left(x_{A}, \vec{p}\right) \cdot\left(1+v_{A} \frac{2 \pi^{2} \gamma^{2} \alpha_{S}\left(\vec{p}^{2}\right) G\left(x_{A}, \vec{p}^{2}\right)}{N_{c} \vec{p}^{2}}+\ldots\right)
$$

Here $\gamma \sim 2$ is the exponent of the large- $\vec{p}^{2}$ behavior of $f(x, \vec{p}) \propto|\vec{p}|^{-2 \gamma}$.

\section{2. $\gamma$-jet production from the breakup $q \rightarrow q \gamma$}

We come to the main point of this talk, namely the associated production of an isolated high $p_{\perp}$ photon and a jet in the proton fragmentation region of $p A$ collisions. Here the production of such photon-jet pairs can be viewed, in the nucleus rest frame, as an an excitation of the $q \gamma$ Fock state of the incident quark, $q^{*} \rightarrow q \gamma$. It is important to realize, that the photon in the $q \gamma$ Fock state doesn't interact, which has the important consequence, that the emerging dijet spectrum becomes a linear functional of the unintegrated nuclear glue.

In the free-nucleon case, where we deal only with a single-gluon exchange at the amplitude level, we obtain the parton-level cross section

$$
\frac{2(2 \pi)^{2} d \sigma_{N}(q \rightarrow q \gamma)}{d z d^{2} \vec{p} d^{2} \vec{\Delta}}=f(x, \vec{\Delta})|\psi(z, \vec{p})-\psi(z, \vec{p}-z \vec{\Delta})|^{2}
$$

with

$$
\left|\psi\left(z, \vec{p}_{1}\right)-\psi\left(z, \vec{p}_{2}\right)\right|^{2}=P_{\gamma q}(z)\left|\frac{\vec{p}_{1}}{\vec{p}_{1}^{2}+\varepsilon^{2}}-\frac{\vec{p}_{2}}{\vec{p}_{2}^{2}+\varepsilon^{2}}\right|^{2}
$$


Here $z, \vec{p}$ are the photon's light-cone momentum fraction and transverse momentum, $\varepsilon^{2}=z m_{q}^{2}$. The decorrelation momentum $\vec{\Delta}=\vec{p}+\vec{p}_{q}=$ measures the deviation of the dijet system from the back-to-back kinematics. Notice that eq.(2.1) is "exact" over the two-body phase-space of the $q-\gamma$ syatem, and contains a contribution from the collinear pole $\vec{p} \sim z \vec{\Delta}$ of the $q \rightarrow q \gamma$ splitting:

$$
\frac{2(2 \pi)^{2} d \sigma_{N}(q \rightarrow q \gamma)}{d z d^{2} \vec{p}}=\underbrace{f\left(x, \frac{\vec{p}}{z}\right)}_{q \rightarrow q} \times \underbrace{\int d^{2} \vec{k}|\psi(z, \vec{k})|^{2}}_{\text {fragmentation } q \rightarrow \gamma}
$$

This contribution must be carefully removed if one is interested in the "dijet"-observables.

One of our main results is the fact that when going to the nuclear target indeed a linear $k_{\perp}-$ factorization "theorem" holds, namely the parton-level cross section has the same form for the nuclear target as it has for the free nucleon target - we only need to swap the proper unintegrated glue. This is in strong contrast to other dijet processes [3, 6]. Per unit transverse area in impact parameter space, the cross section reads:

$$
\frac{(2 \pi)^{2} d \sigma_{A}(q \rightarrow q \gamma)}{d z d^{2} \vec{p} d^{2} \vec{\Delta} d^{2} \vec{b}}=\phi\left(v_{A}, x, \vec{\Delta}\right)|\psi(z, \vec{p})-\psi(z, \vec{p}-z \vec{\Delta})|^{2}+w_{0}\left(v_{A}\right) \delta^{(2)}(\vec{\Delta})|\psi(z, \vec{p})-\psi(z, \vec{p}-z \vec{\Delta})|^{2}
$$

Here we indicated a potentially factorization-violating term, which comes from diffractive dissociation of the quark. It vanishes due to the $\delta$-function-like $\vec{\Delta}$-shape of nuclear diffraction. This result serves to prove that $\gamma$-jet production is a linear probe of the nuclear unintegrated glue.

Interestingly a simple analytical result can be obtained for the nuclear broadening of the acoplanarity-momentum distribution as quantified by

$$
\left\langle\vec{\Delta}^{2}\right\rangle_{A-N}=\frac{\left\langle\vec{\Delta}^{2} d \sigma\right\rangle_{A}-\left\langle\vec{\Delta}^{2} d \sigma\right\rangle_{N}}{T(\vec{b}) \int d^{2} \vec{\Delta}\left(d \sigma_{N} / d^{2} \vec{p} d^{2} \vec{\Delta}\right)}
$$

Using (1.6) and carefully removing the collinear pole from the $\gamma$-jet spectrum, we obtain

$$
\left\langle\vec{\Delta}^{2}\right\rangle_{A-N} \approx \frac{2 \pi^{2} \gamma^{2}}{N_{c}} \alpha_{S}\left(\vec{p}^{2}\right) G\left(x_{A}, \vec{p}^{2}\right) T(\vec{b}) \approx Q_{A}^{2}(\vec{b}, x) \cdot \frac{\gamma^{2}}{2} \cdot \frac{\alpha_{S}\left(\vec{p}^{2}\right) G\left(x_{A}, \vec{p}^{2}\right)}{\alpha_{S}\left(Q_{A}^{2}\right) G\left(x_{A}, Q_{A}^{2}\right)} .
$$

This means that the nuclear broadening of the acoplanarity-momentum distribution directly measures the nuclear saturation scale $Q_{A}^{2}$. Nuclear effects are conveniently visualized by the ratio (we stay at the parton-level)

$$
R_{p A}\left(v_{A}, \vec{p}, \vec{\Delta}\right)=\frac{d \sigma_{A}}{T_{A}(\vec{b}) d \sigma_{N}}=\frac{\phi\left(v_{A}, x, \vec{\Delta}\right)}{v_{A} f(x, \vec{\Delta})}
$$

And similarly the central-to-peripheral ratio, which involves only nuclear quantities:

$$
R_{c p}\left(v_{>}, v_{<}, \vec{p}, \vec{\Delta}\right)=\frac{v_{<} \phi\left(v_{>}, x, \vec{\Delta}\right)}{v_{>} \phi\left(v_{<}, x, \vec{\Delta}\right)}
$$

Both these ratios do not depend on the photon's transverse momentum $\vec{p}$. We show the ratio $R_{p A}$ at $x=x_{A}$ for different opacities in the right panel of fig. 目. We observe a shadowing at small values of $\vec{\Delta}$ and a Cronin-type peak which position reflects the $v_{A}$-dependent saturation scale. In the left panel we show $R_{c p}$ and its evolution with $x$. While it displays the same Cronin-peak as $R_{p A}$ for $x=x_{A}$, the latter is entirely quenched at small $x$. 

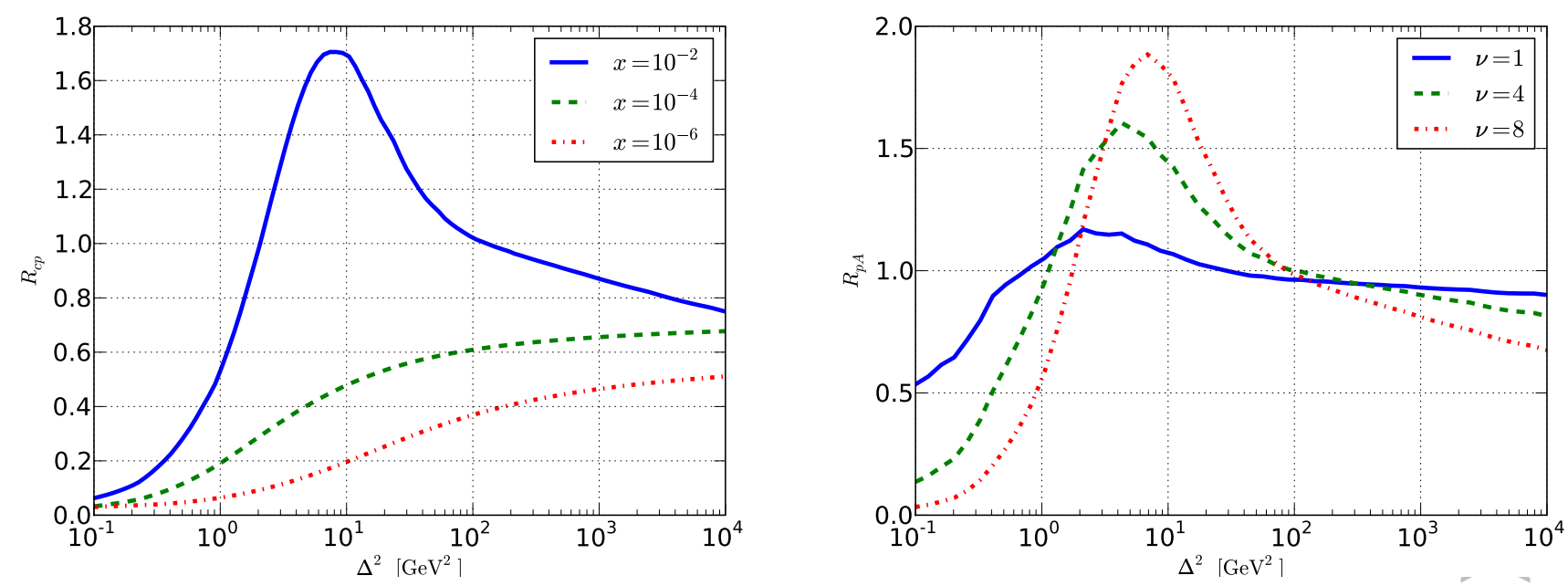

Figure 2: Left: $R_{c p}$ for $v_{>}=8, v_{<}=1$ for different $x$; Right: $R_{p A}$ at $x=0.01$.

\section{Summary}

In summary, hard isolated photon production in the proton fragmentation region of $p A$ collisions is a good probe of the nuclear unintegrated gluon distribution. Relevant cross sections linearly depend on the nuclear unintegrated gluon distribution.

The nuclear broadening of the acoplanarity distribution (at least at not too small $x$ ) is directly proportional to the saturation scale.

\section{Acknowledgements}

The results presented here have been obtained in collaboration with Kolya Nikolaev. I would like to thank Antoni Szczurek for reliable transport Kraków-Florence.

\section{References}

[1] N. N. Nikolaev, B. G. Zakharov and V. R. Zoller, Z. Phys. A 351 (1995) 435.

[2] N. N. Nikolaev, W. Schäfer, B. G. Zakharov and V. R. Zoller, JETP Lett. 84 (2007) 537.

[3] N. N. Nikolaev, W. Schäfer, B. G. Zakharov and V. R. Zoller, J. Exp. Theor. Phys. 97 (2003) 441.

[4] N. N. Nikolaev, W. Schäfer and B. G. Zakharov, Phys. Rev. Lett. 95 (2005) 221803.

[5] N. N. Nikolaev, W. Schäfer and G. Schwiete, Phys. Rev. D 63 (2001) 014020.

[6] N. N. Nikolaev, W. Schäfer, B. G. Zakharov and V. R. Zoller, Phys. Rev. D 72 (2005) 034033;

N. N. Nikolaev, W. Schäfer and B. G. Zakharov, Phys. Rev. D 72 (2005) 114018. 